\title{
Comparison of Mannitol and Meglumine Diatrizoate for Stomach and Small Bowel Wall Study by Computed Tomography, a Prospective Study
}

\begin{abstract}
Nikhil Vikram ${ }^{1}$, Anand M Rahalkar ${ }^{2}$, Mukund Rahalkar ${ }^{3}$, Akhil Halkude $^{4}$, Priyanka Rana ${ }^{5}$, Priyanka Khopade
${ }^{1}$ Fellow, Chikitsa Ultrasound Training and Research Centre, Chembur, Mumbai, 400071, ${ }^{2}$ Associate Professor, Department of Radiology, Bharati Vidyapeeth (Deemed to be University) Medical College, Pune, Dhankawadi, Pune 400043, ${ }^{3}$ Ex Professor, Department of Radiology, Bharati Vidyapeeth (Deemed to be University) Medical College. Dhankawadi, Pune 400043, ${ }^{4}$ Consultant Radiologist, Ashwini Sahakari Rugnalaya, Solapur, ${ }^{5}$ Senior Resident, Bharati Vidyapeeth (Deemed to be University) Medical College, Pune, Dhankawadi, Pune 400043, ${ }^{6}$ Senior Resident, Bharati Vidyapeeth (Deemed to be University) Medical College, Pune, Dhankawadi, Pune 400043, India
\end{abstract}

Corresponding author: Dr Anand M Rahalkar, Associate Professor, Department of Radiology, Bharati Vidyapeeth (Deemed to be University) Medical College, Pune, Dhankawadi, Pune 400043, India

DOI: http://dx.doi.org/10.21276/ijcmsr.2019.4.3.35

How to cite this article: Nikhil Vikram, Anand M Rahalkar, Mukund Rahalkar, Akhil Halkude, Priyanka Rana, Priyanka Khopade. Comparison of mannitol and meglumine diatrizoate for stomach and small bowel wall study by computed tomography, a prospective study. International Journal of Contemporary Medicine Surgery and Radiology. 2019;4(3):C162-C167.

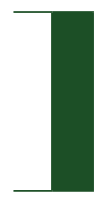

\section{A B S T R A C T}

Introduction: Mannitol, also known as mannite or manna sugar, is a white, crystalline solid that looks and tastes sweet like sucrose. The main purpose of this study was to analyze the use of Mannitol and Meglumine Diatrizoate on patients undergoing CT abdomen for quantitative and qualitative comparison of small bowel enhancement.

Materials and Methods: A total of 100 consecutive patients referred to Radio diagnosis department between November 2018 and July 2019 for 16 slice MDCT of the abdomen and pelvis with intravenous contrast enhancement were randomly selected. Patients will be divided into two groups comprising 50 patients each receiving 3\% Mannitol and Meglumine Diatrizoate. Patients were selected on the basis of inclusion and exclusion criteria. A case record proforma was prepared for each patient. Each subject received $1200 \mathrm{ml}$ of oral contrast. Group 1 received 3\% mannitol in water and group 2 received diluted iodinated positive contrast.

Results: Quantitative analysis showed statistical significant $(p<0.001)$ superiority of Mannitol for bowel wall delineation. Qualitative analysis for bowel distension, fold visibility, intraluminal homogeneity, artifacts and overall image quality show better cores with Mannitol. Interobserver agreement between two radiologist ranges from 0.491 to 0.95.

Conclusion: Mannitol as endoluminal contrast increases the diagnostic accuracy of the investigative studies in comparison to iodine-based contrast by producing significantly better visibility of mural features with improved image quality without additional adverse effects.

Keywords: Mannitol, Bowel Wall, Bowel Lumen Attenuation.

\section{INTRODUCTION}

Protocols for abdominal CT imaging traditionally include the administration of intravenous contrast material and the use of oral contrast media for bowel delineation through distension and opacification. As collapsed sections of bowel can falsely mimic bowel wall thickening, adequate distension of the bowel wall is required for assessment of the wall thickness and better visualization of wall characteristics. Adequate opacification of the bowel can be achieved by the administration of oral contrast agents which facilitate discrimination of bowel from other soft tissue by providing adequate bowel distension and optimal contrast between the bowel wall and the lumen., ${ }^{1,2-8}$ Oral contrasts depend on structural changes in the bowel to demonstrate pathological lesions and can either be negative, neutral or positive. Negative oral contrasts include low-attenuation contrast agents such as air and carbon dioxide. Neutral oral contrasts are those having an attenuation value similar to that of water (10-30 HU) for e.g. Milk, Juice, Low density barium (VoLumen), Polyethylene glycol, Lactulose, SCC, Locust bean gum and Mannitol. Positive OCM include dilute barium solutions and diluted water soluble iodinated contrast media like Meglumine Diatrizoate, and are most commonly used as the standard GIT distension method. ${ }^{1}$

Neutral oral contrast agents like Mannitol have proved to be effective in the diagnosis of Crohn"s disease ${ }^{3,4}$, neoplasms ${ }^{5}$ and bowel-ischemia. ${ }^{6}$ They are also used to distend and delineate the stomach and duodenum in imaging of pancreatic and biliary disease. ${ }^{7}$ The most widely used neutral contrast agent is water. It has excellent contrast behavior in the upper GIT. Its clinical use for the distal parts of the small bowel, however, is 
limited due to its rapid absorption. ${ }^{4,7}$ Additives can slow the absorption of water by increasing the osmolarity. ${ }^{3}$ One such additive is Mannitol, an inexpensive and easy-to-use neutral oral contrast agent. Many studies suggest that Mannitol provide better distension and visualization of gastrointestinal system. ${ }^{4,7}$

From the viewpoint of visibility on contrast enhanced CT without high attenuation oral contrast material, the mucosa, sub mucosa, and muscularis externa are most clearly distinguished if sub mucosal fat, edema, or hemorrhage is abundant enough to result in a three layer appearance (target sign) of the intestinal wall. ${ }^{9}$

Current research aimed to study the stomach and small bowel anatomy on MDCT after oral contrast administration and side effect of Mannitol and Meglumine Diatrizoate in both groups and to analyze small bowel enhancement qualitatively and quantitatively in both groups.

\section{MATERIAL AND METHODS}

Study was done on the patients who were referred to the Department of Radio diagnosis, Bharati Medical College, Dhankawadi, Pune, for Pain in abdomen, infection and abdominal mass. Study was done for the period of 9 months.

Exclusion Criteria: Patients with history of allergy to any iodinated contrast. Age and sex matching was done and the subjects were of age 20-70 years. The neutral oral contrast group was given a 3\% Mannitol and the positive oral contrast group was given Lekgraf (Meglumine Diatrizoate).

Administration of the contrast agents was standardized. All patients consumed $1200 \mathrm{ml}$ of endoluminal contrast agent; $1000 \mathrm{ml}$ of endoluminal contrast was consumed over a time period of 30-45 min and the remaining $200 \mathrm{ml}$ at $10 \mathrm{~min}$ before the scan. For standardization and uniform distension of small bowel, patients were asked to drink $100 \mathrm{ml}$ every 4-5 min for 45-55 min. Imaging was performed 55-60 min after the beginning of contrast agent consumption.

First group received neutral oral contrast, 3\% Mannitol in 1,200 $\mathrm{ml}$ of tap water mixed with orange squash as a sweetening agent.

Second group received positive oral contrast, Lekgraf (Meglumine Diatrizoate) $20 \mathrm{ml}$ in $1200 \mathrm{ml}$ of tap water mixed with orange squash as a sweetening agent.

\section{Scanning Protocol}

All examinations were performed on a 16-detector row MDCT scanner (Philips). Using a power injector, 80 to100 $\mathrm{ml}$ of intravenous contrast was administered according to patient size, i.e. approximately $1.5 \mathrm{ml}$ per $\mathrm{kg}$ of body weight at a concentration of $300 \mathrm{mg} / \mathrm{ml}$ iodine (Omnipaque 300) followed by a saline flush of $40 \mathrm{ml}$ at the same flow rate. The flow rates ranged between 1.5 and $4 \mathrm{ml} / \mathrm{s}$. This variation was a result of difficult venous access in some patients.

Bolus tracking method was used for acquisition of arterial and portal venous phases with a delay of $8 \mathrm{~s}$ post threshold achievements in lower thoracic aorta for arterial phase and a delay of $45 \mathrm{~s}$ post-threshold for portal venous phase. Images were reconstructed in axial and coronal planes with a slice thickness and interval of $5 \mathrm{~mm}$. All Images were then sent to Picture Archiving and Communication System (PACS) for analysis.

Two experienced radiologists independently reviewed the images on Picture Archiving and Communication System (PACS). Both reviewers were unaware to the clinical history.

\section{Qualitative analysis}

An incremental three-point scale ( 0 =fair, $1=$ good and $2=$ excellent) was used to rate images from each examination for bowel distension, homogeneity of the lumen, delineation of the bowel wall, presence of artifacts and overall image quality using only CT images. Score 0 -fair $(<25 \%$ of the bowel loops show adequate distension or homogeneity of luminal contents or fold visibility), score $1-\operatorname{good}(25-75 \%$ of the bowel loops show adequate distension or homogeneity of luminal contents) and score 2-excellent (75-100\% of the bowel loops show adequate distension or homogeneity of luminal contents or fold visibility). The presence of artifacts was scored from no artifacts (0) to serious image degradation due to artifacts (4) and overall image quality from unreadable (0) to perfect (4). Maximum distension of stomach, pylorus, and small bowel loops was measured by taking outer to outer wall dimension.

Quantitative analysis: The maximum cross-sectional diameter of the antrum of the stomach, horizontal part of the duodenum, proximal jejunum, and terminal ileum were measured perpendicular to the axis of the lumen using the outer margins of the intestinal wall for each patient Attenuation [in Hounsfield units (HU)] of the lumen and small bowel wall were measured at the same levels of the stomach, duodenum, jejunum, and terminal ileum. Attenuation of the lumen was measured by placing a region of interest (ROI) within a well-distended segment of the small bowel section.

Attenuation of the wall was measured by first zooming into the image section until the wall is clearly visible and then placing an ROI over the bowel wall.

For each measured section of the GIT, the contrast for the bowel wall was calculated as the difference in attenuation values (contrast) of the lumen and wall by subtracting the $\mathrm{HU}$ value obtained from the lumen from the HU value obtained from the corresponding gastrointestinal wall.

\section{STATISTICAL ANALYSIS}

The Mann-Whitney U-test was used to perform statistical analysis of the Differences in median scores between the neutral oral contrast and positive oral contrast groups regarding bowel distension, homogeneity of the lumen, differentiation of the bowel wall against luminal content and surrounding fat, the presence of artifacts and overall image quality. Differences were considered significant if $\mathrm{P}<0.05$. Inter-observer agreement was evaluated using linear-weighted kappa statistics. Regarding the quantitative analysis, the differences in maximum diameters, HU values for the GIT lumen, and contrast values between the neutral oral contrast and positive oral contrast groups were compared using Student's unpaired t-test. Differences were considered significant if $\mathrm{P}<0.05$. 


\section{RESULTS}

Excellent and uniform small bowel distension with good small bowel wall/fold visualization and homogeneity of intraluminal content is achieved by mannitol (Figure-1). Less bowel distension, poor fold visibility and increased luminal attenuation as compared to mannitol is noted in patient prepared with meglumine diatrazoate (Figure-2). Figure 3 reveals symmetric concentric mural thickening of terminal ileum in right iliac fossa suggestive of Koch's disease.

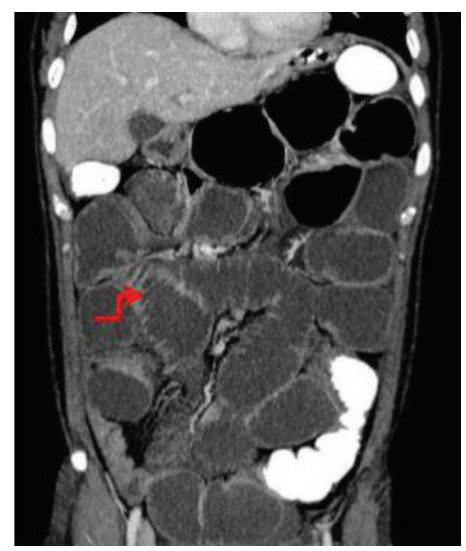

Figure-1: Coronal CT shows excellent and uniform small bowel distension with good small bowel wall/fold visualization and homogeneity of intraluminal content achieved by mannitol.

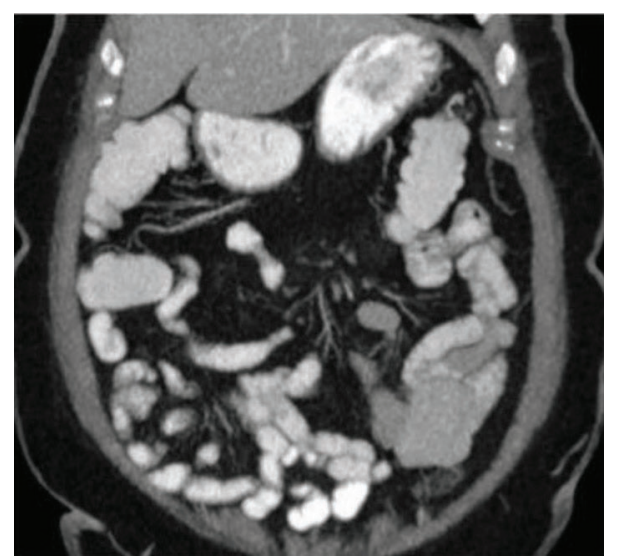

Figure-2: Coronal CT shows less bowel distension, poor fold visibility and increased luminal attenuation as compared to mannitol in patient prepared with meglumine diatrazoate.
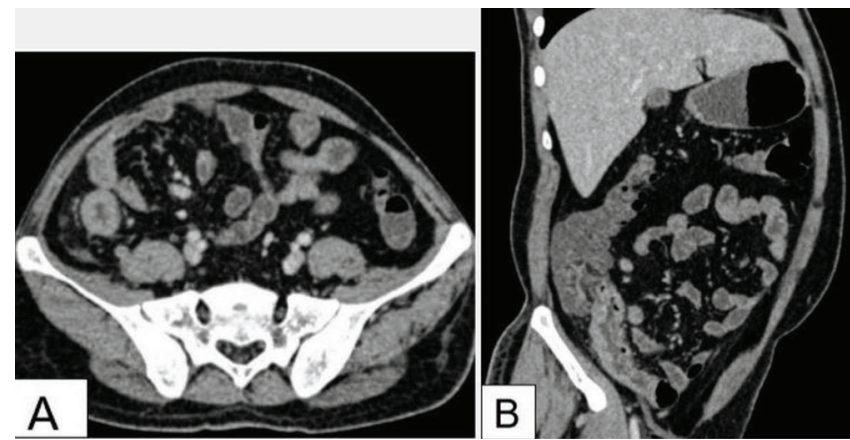

Figure-3: Axial CT (A) and Coronal CT (B) shows symmetric concentric mural thickening of terminal ileum in right iliac fossa suggestive of Koch's disease.
Figure 4 shows focal thickening and hyperdense wall of a jejunal loop, cross section of bowel wall shows three layers of high (outer), low (middle), and high (inner) attenuation. Together, these layers create a target appearance known as the target sign. Figure 5 shows smooth long segment narrowing of ileum to suggest stricture with proximal small bowel dilatation. Figure 6 shows heterogeneously enhancing soft tissue deposits in peritoneum and mesentery and two distinct areas of small bowel intussusception. Coronal CT image shows smooth long segment narrowing of ileum to
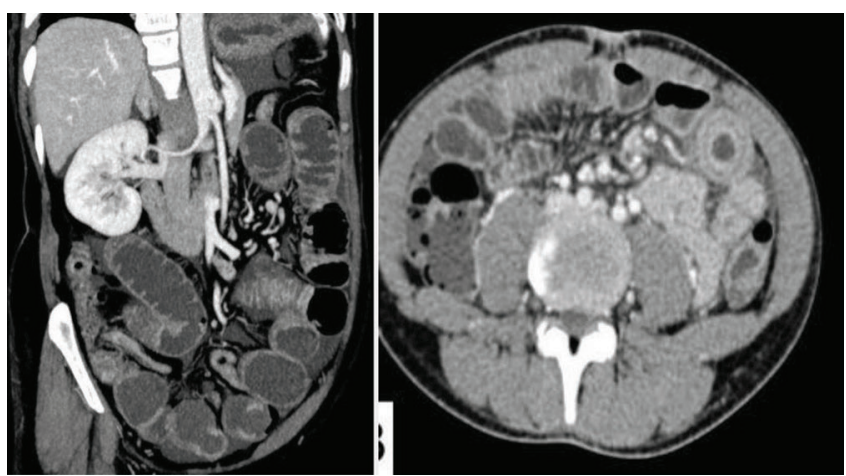

Figure-4 - Coronal CT (A) and axial CT (B) shows focal thickening and hyperdense wall of a jejunal loop, cross section of bowel wall shows three layers of high (outer), low (middle), and high (inner) attenuation. Together, these layers create a target appearance known as the target sign.

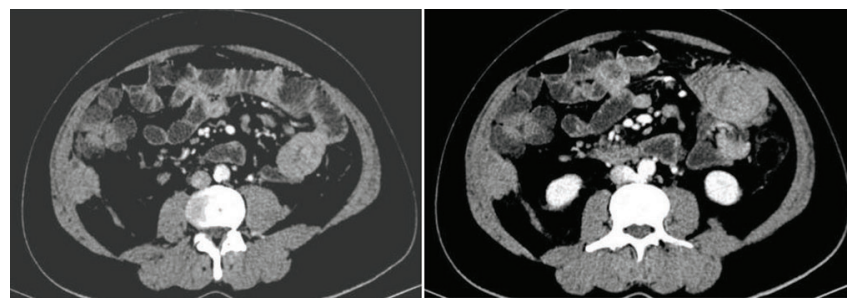

Figure-5: Coronal CT image shows smooth long segment narrowing of ileum to suggest stricture with proximal small bowel dilatation

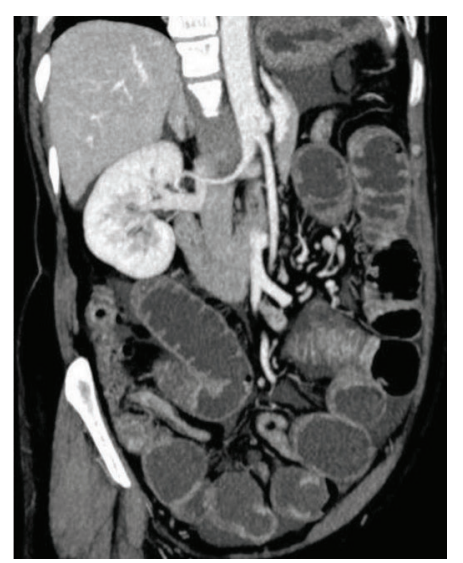

Figure-6: Axial CT images shows heterogeneously enhancing soft tissue deposits in peritoneum and mesentery. Two distinct areas of small bowel intussusception is noted. Coronal CT image shows smooth long segment narrowing of ileum to suggest stricture with proximal small bowel dilatation 

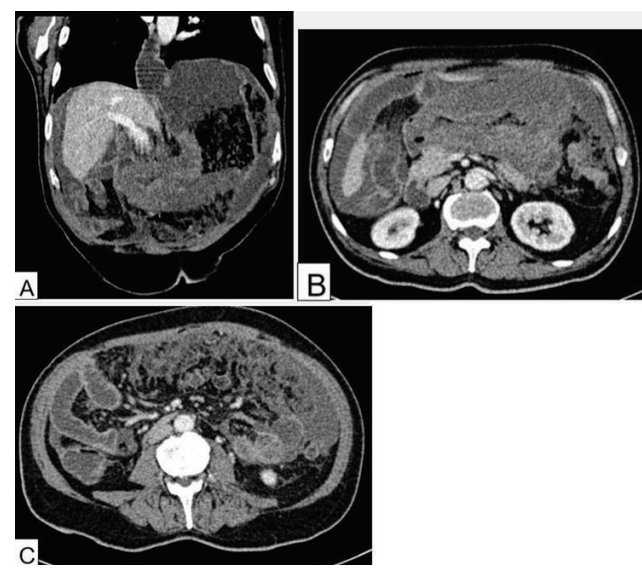

Figure-7: Coronal CT (A) and Axial CT (B) images shows marked thickening of walls of body and antrum with marked narrowing of lumen in a follow up case of Carcinoma stomach.CT axial image (C) show omental thickening
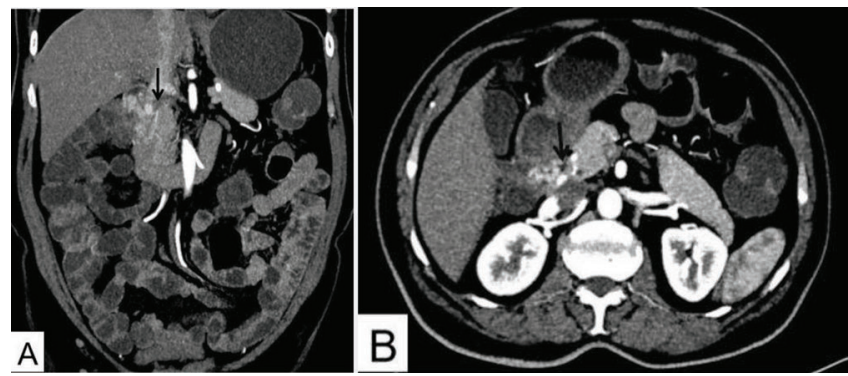

Figure-8: Coronal CT (A) and axial CT (B) images shows multiple tiny enhancing foci of enhancement in second part of duodenum with Mannitol as oral contrast suggestive of deposits from neuroendocrine tumor.
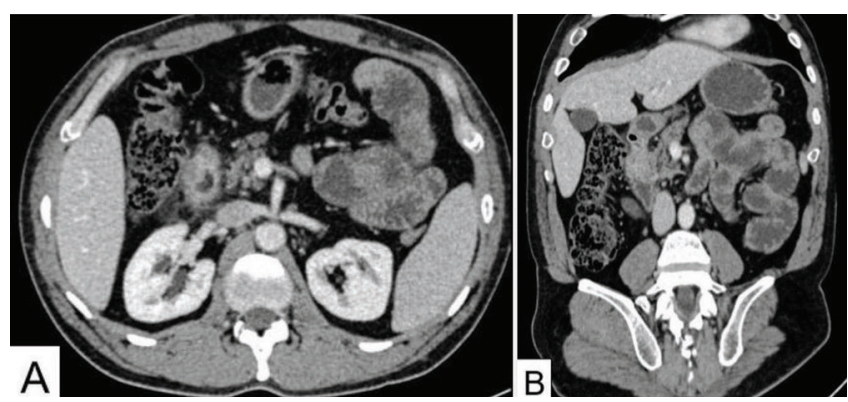

Figure-9: Axial CT (A) and Coronal CT (B) images show thickened and enhancing walls of second part of duodenum, suggestive of Duodenitis

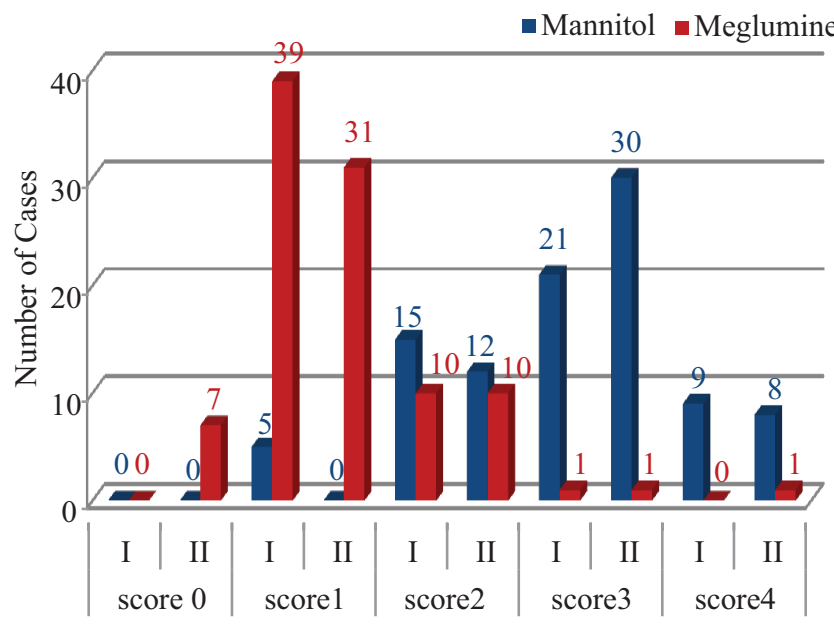

Qualitative Analysis of Overall Image Quality

Figure-10: Comparative bar diagram for Qualitative Analysis of Overall Image Quality.

\begin{tabular}{|l|l|c|c|c|c|}
\hline Location & Oral contrast & $\begin{array}{c}\text { Diameter }(\mathbf{c m}) \pm \\
\text { Standard Deviation }\end{array}$ & $\begin{array}{c}\text { Attenuation of } \\
\text { bowel wall (HU) } \pm \\
\text { Standard Deviation }\end{array}$ & $\begin{array}{c}\text { Attenuation of } \\
\text { bowel lumen (HU) } \pm \\
\text { Standard Deviation }\end{array}$ & $\begin{array}{c}\text { Difference wall to } \\
\text { lumen (HU) } \pm \\
\text { Standard Deviation }\end{array}$ \\
\hline \multirow{2}{*}{ Stomach } & Mannitol & $4.25 \pm 0.89$ & $100.5 \pm 19.7$ & $17.5 \pm 12.8$ & $84 \pm 19.33$ \\
\cline { 2 - 6 } & Meglumine & $4.2 \pm 0.83$ & $91 \pm 30.27$ & $96 \pm 45.7$ & $-6.5 \pm 56.67$ \\
\hline \multirow{2}{*}{${ }^{\text {rd }}$ part of duodenum } & Mannitol & $2.1 \pm 0.69$ & $100 \pm 23.27$ & $24.5 \pm 27.98$ & $64.5 \pm 36.14$ \\
\cline { 2 - 6 } & Meglumine & $2.0 \pm 0.44$ & $91 \pm 23.94$ & $90.5 \pm 66.79$ & $1.5 \pm 76.23$ \\
\hline \multirow{2}{*}{ Jejunum } & Mannitol & $2.4 \pm 0.89$ & $98.5 \pm 19.87$ & $20 \pm 26.07$ & $74 \pm 27.92$ \\
\cline { 2 - 6 } & Meglumine & $2.2 \pm 0.39$ & $107.5 \pm 29.58$ & $93 \pm 48.85$ & $6.5 \pm 46.31$ \\
\hline \multirow{2}{*}{ Terminal ileum } & Mannitol & $1.85 \pm 0.44$ & $90.5 \pm 19.11$ & $15 \pm 22.63$ & $67 \pm 31.26$ \\
\cline { 2 - 6 } & Meglumine & $1.7 \pm 0.40$ & $100 \pm 38.84$ & $264 \pm 175.59$ & $-148 \pm 170.79$ \\
\hline
\end{tabular}

Table-1: Comparative table for quantitative analysis of bowel distension and attenuation (in $\mathrm{cm}$ with Standard Deviation) of bowel loops at various levels.

\begin{tabular}{|l|c|c|c|c|c|c|}
\hline Score & \multicolumn{2}{|c|}{ Fair (score=0) } & \multicolumn{2}{c|}{ Good (score=1) } & \multicolumn{2}{c|}{ Excellent (score=2) } \\
\hline Observer Contrast & $\mathrm{I}$ & $\mathrm{II}$ & $\mathrm{I}$ & $\mathrm{II}$ & $\mathrm{I}$ & $\mathrm{II}$ \\
\hline Mannitol & 2 & 0 & 22 & 8 & 26 & 42 \\
\hline Meglumine & 38 & 29 & 12 & 17 & 0 & 4 \\
\hline \multicolumn{6}{|c|}{ Table-2: Comparative table for Qualitative Analysis of Bowel Distension } \\
\hline
\end{tabular}

\begin{tabular}{|l|c|c|c|c|c|c|}
\hline Score & \multicolumn{2}{|c|}{ Fair (score=0) } & \multicolumn{2}{c|}{ Good (score=1) } & \multicolumn{2}{c|}{ Excellent (score=2) } \\
\hline Observer Contrast & $\mathrm{I}$ & $\mathrm{II}$ & $\mathrm{I}$ & $\mathrm{I}$ & $\mathrm{I}$ & $\mathrm{I}$ \\
\hline Mannitol & 3 & 0 & 19 & 5 & 28 & 45 \\
\hline Meglumine & 30 & 14 & 20 & 33 & 0 & 3 \\
\hline \multicolumn{7}{|c|}{ Table-3: Comparative table for Qualitative Analysis of Fold Visibility } \\
\hline
\end{tabular}


suggest stricture with proximal small bowel dilatation. Figure 7 shows marked thickening of walls of body and antrum with marked narrowing of lumen in a follow up case of Carcinoma stomach. CT axial image (C) show omental thickening. Figure 8 shows multiple tiny enhancing foci of enhancement in second part of duodenum with Mannitol as oral contrast suggestive of deposits from neuroendocrine tumor. Figure 9 show thickened and enhancing walls of second part of duodenum, suggestive of Duodenitis.

\section{DISCUSSION}

Age Distribution among Study Population shows that maximum number of patients (34\% of the cases) belongs to age group of 61-70 years in both case groups, followed by $18 \%$ and $26 \%$ of the cases belonging to $41-50$ years in Group 1 and Group 2 respectively.

In a study done by Megibow AJ, Babb JS, Hecht EM et $\mathrm{al}^{7}$ with a study group of 60 patients mean age of patients taking VoLumen is 58.1 years and those taking water with methylcellulose is 57.4 years.

Sex distribution among study population reveals 26 patients (52.0\% of the cases) were male and 24 patients $(48.0 \%$ of the cases) were female in Group 1 and 10 patients $(20.0 \%$ of the cases) were male and 40 patients ( $80.0 \%$ of the Cases) were female in Group 2.

In a study done by Megibow AJ, Babb JS, Hecht EM et $\mathrm{al}^{7}$ with a study group of 60 patients, 33 were female (55\%)and 27 (45\%) were male.

Table 1 reveals that Mannitol shows higher median scores than Meglumine for bowel distension in each location of the bowel, however, difference between scores were not statistically significant $(\mathrm{P}>.05)$. Jejunum and Stomach shows statistically significant difference in attenuation of bowel wall $(p<0.5)$. There is statistically significant difference in median scores of attenuation of lumen and attenuation difference from wall to lumen at all four locations $(\mathrm{P}<0.5)$.

In a study done by K Prakashini, Chandan Kakkar, Charudutt Sambhaji et $\mathrm{al}^{8}$, did a similar study on 300 patients no statistical significant difference in distension of stomach and duodenum was found between the three groups. Mean distension of jejunum and ileum shows statistically significant difference and measures $1.97 \mathrm{~cm}$ and $2.1 \mathrm{~cm}$ respectively with Mannitol and 1.70 and $1.71 \mathrm{~cm}$ with Meglumine Diatrizoate. Table 2 reveals better bowel distension by Mannitol as compared to meglumine. Score=2 (Excellent) by observer I with Mannitol was 26 and Meglumine was 0 , by observer II with Mannitol was 42 and Meglumine was 4. In a study done by K Prakashini, Chandan Kakkar, Charudutt Sambhaji et al ${ }^{8}$, the qualitative analysis of overall bowel distension. Score $=0$ (Fair) with Mannitol was 10 and Meglumine was 56, Score $=2$ (Excellent) with Mannitol was 26 and Meglumine was 8.

Table 3 reveals better fold visibility by mannitol as compared to meglumine. The qualitative analysis of fold visibility of Score $=2$ (Excellent) by observer I with Mannitol was 28 and Meglumine was 0, by observer II with Mannitol was 45 and Meglumine was 3. In a study done by K Prakashini, Chandan Kakkar, Charudutt Sambhaji et $\mathrm{al}^{8}$, the qualitative analysis of fold visibility. Score=0 (FAIR) with Mannitol was 8 and Meglumine was 43, Score=2 (Excellent) with Mannitol was
34 and Meglumine was 0.

Figure 10 shows qualitative analysis of overall image quality:

1) Score $=0$ (unreadable) by observer I with both Mannitol and Meglumine was 0, by observer II with Mannitol was 0 and Meglumine was 7.

2) Score=1 (fair) by observer I with Mannitol was 5 and Meglumine was 39, by observer II with Mannitol was 0 and Meglumine was 31.

3) Score $=2$ (good) by observer I with Mannitol was 15 and Meglumine was 10, by observer II with Mannitol was 12 and Meglumine was 10 .

4) Score=3 (nearly perfect) by observer I with Mannitol was 21 and Meglumine was 1, by observer II with Mannitol was 30 and Meglumine was 1 .

5) Score $=4$ (perfect) by observer I with Mannitol was 9 and Meglumine was 0 by observer II with Mannitol was 8 and Meglumine was 1 .

In our study, interobserver agreement for overall image quality by SPSS Statistics software, Kappa $\kappa=0.678$. It suggest fair to good interobserver agreement. Interobserver agreement in our study ranges 0.491 to 0.95 .

In a study done by Berther R, Patak MA, Eckhardt B et $\mathrm{al}^{4}$, Interobserver agreement in our study ranges 0.44 to 0.67 .

Side effects with Mannitol and Meglumine: Both contrast agents were well tolerated by the patients and considered the taste of Mannitol as fine and suitable without any discomfort. No major complications were reported by clinicians. Three patients reported a mild frequency of watery stool following Mannitol intake. In a study done by Berther R, Patak MA, Eckhardt $\mathrm{B}$ et $\mathrm{al}^{4}$, all patients tolerated oral contrast very well and no side effect is noted.

\section{CONCLUSION}

MDCT of abdomen and pelvis with 3\% Mannitol as neutral oral contrast is simple, non-invasive and effective method of evaluating small bowel disease which lead to good overall image quality with better visualization of mural features of the small bowel and homogeneous image. Consistent rate of oral intake is very important for Intraluminal homogeneity. Quantitative analysis shows statistically significant ( $p<$ 0.001) superiority of Mannitol for bowel wall delineation. Qualitative analysis for bowel distension, fold visibility, intraluminal homogeneity, artifacts and overall image quality show better cores with Mannitol.

\section{REFERENCES}

1. Erica Lauren Buttigieg, Karen Borg Grima, Kelvin Cortis, Sandro Galea Soler, Francis Zarb. An evaluation of the use of oral contrast media in abdominopelvic CT. Eur Radiol 2014;24:2936-2944.

2. Rakesh Sinha. Recent advances in intestinal imaging. Indian J Radiol Imaging. 2011; 21(3): 170-175

3. Zhang LH, Zhang SZ, Hu HJ, Gao M, Zhang M, Cao Q, Zhang QW. Multidetector CT enterography with iso-osmotic mannitol as oral contrast for detecting small bowel disease. World J Gastroenterol. 2005;11(15):2324-9.

4. Berther R, Patak MA, Eckhardt B, Erturk SM, Zollikofer CL. Comparison of neutral oral contrast 
versus positive oral contrast medium in abdominal multidetector CT. Eur Radiol. 2008

5. Horton KM, Fishman EK. Multidetector- row computed tomography and 3dimensional computed tomography imaging of small bowel neoplasms: current concept in diagnosis. J Comput Assist Tomogr 2004;28(2):106-116.

6. Seong Sook Hong, Ah Young Kim, Jae Ho Byun et al. MDCT of Small-Bowel Disease: Value of 3D Imaging American Journal of Roentgenology. 2006;187: 12121221. ep;18(9):1902-9.

7. Megibow AJ, Babb JS, Hecht EM et al. Evaluation of bowel distention and bowel wall appearance by using neutral oral contrast agent for multidetector row CT. Radiology 2006;7(3):87-95.

8. K Prakashini, Chandan Kakkar, Charudutt Sambhaji, Chandrakant M Shetty, Vedula Rajanikanth Rao. Quantitative and qualitative bowel analysis using mannitol, water and iodine-based endoluminal contrast agent on 64-row detector CT. Indian J Radiol Imaging. 2013; 23(4): 373-378.

9. Chung Kuao Chou. CT manifestations of small bowel ischemia due to impaired venous drainage-with a correlation of pathologic findings. Indian J Radiol Imaging. 2016; 26(3): 342-351.

Source of Support: Nil; Conflict of Interest: None

Submitted: 13-07-2019; Accepted: 07-08-2019; Published online: 09-09-2019 